open access wWW.ijecs.in

International Journal Of Engineering And Computer Science

Volume 9 Issue 09 September 2020, Page No. 25156-25160

ISSN: 2319-7242 DOI: 10.18535/ijecs/v9i09.4524

\title{
A Peek into Theory of Automation with Applications to Various Branches of Science
}

\author{
Divyalaxmi $\mathbf{N}$.
}

Potti Sriramulu Nellore, Andhra Pradesh, India

\begin{abstract}
:
In this paper, we shall be concerned with the existence and uniqueness of solution to three- point boundary value problems associated with a system of first order matrix difference system. Shortest and Closest Lattice vector methods are used as a tool to obtain the best least square solution of the three-point boundary value problems when the characteristic matrix $\mathrm{D}$ is rectangular.
\end{abstract}

Keywords: Coronavirus, COVID-19, Distribution Probability, Genome Sequence.

\section{Introduction}

Let's start our discussion with Automata theory, which in simple theory is the study of abstract machines and the term automata comes from Greek word and it means "Self- acting". Moreover, automata play a major role in the theory of computation, compiler design, artificial intelligence, parsing and formal verification. Further, the success of theoretical computer science is the fact that real computers can be modeled with very simple abstract machines. The model infact ignore the implementation details of individual computers and concentrate on the actual computational aspects. Automata theory is the basis for formal languages. Formal languages are normally defined in one of the three ways and they can be described in automata theory as Regular Expressions or Standard Automata or a grammar system. Grammar automata can produce a wide variety of complex languages from a very few symbols and very few productions rules. Our main aim in this paper is to produce complex languages by using typical symbols and few production rules and show some applications in mathematical biology and in cell biology.

Definition1.1. an alphabet $\sum$ is any finite set. We often write

$$
\Sigma=\left\{a_{1}, a_{2}, \ldots \ldots, a_{n}\right\}
$$

They $a_{i}$ are called the symbols of the alphabet set.

A string is a finite sequence of symbols. Technically it is more convenient to define strings as functions.

For any integer $\mathrm{n}>1$, let

$$
[n]=\{1,2, \ldots \ldots \ldots, n\} \text {. }
$$

For $\mathrm{n}=0$, let

$$
[0]=\varphi \text {, an empty set. }
$$

Definition 1.2: Given an alphabet set $\sum$, a string over $\sum$ of length $n$ is any function say

$$
u:[n] \rightarrow \Sigma \text {. }
$$

The integer $n$ is the length of the string $\mu$ and it is denoted as $|\mu|$. then $n=0$ the special string

$$
u:[0] \rightarrow \Sigma
$$

of length 0 is called the empty string or null string, and is denoted as $\varepsilon$.

Given a string $u:[n] \rightarrow \Sigma$ of length $n \geq 1, u(i)$ denotes the $i^{\text {th }}$ letter of the string $u$. For simplicity of notation, we denote the string $\mathrm{u}$ as

$$
\mathrm{u}=\mathrm{u}_{1} \mathrm{u}_{2} \ldots \mathrm{u}_{\mathrm{n}}
$$

with each $\mathrm{u}_{\mathrm{i}} \in \Sigma$. For example, if $\Sigma=\{a, b, c\}$ and $u:[4] \rightarrow \Sigma$ is defined as 
$u(1)=b, u(2)=a, u(3), u(4)=b$,

then

$u=b a c b$.

Strings of length 1 are functions : [1] $\rightarrow \Sigma$. Simply picking some element $\mathrm{u}(1)=a_{i}$ in $\Sigma$. Thus, we will identify every symbol $a_{i} \in \Sigma$ with the corresponding string of length 1.

The set of all strings over an alphabet set $\Sigma$, including the empty string is denoted by $\Sigma^{*}$.

Concatenation: The binary operation on $\Sigma^{*}$ is defined as follows:

If $a_{1} a_{2} \ldots a_{n} \in \Sigma^{*}$ and $b_{1} b_{2} \ldots b_{m} \in \Sigma^{*}$, then the concatenation

$$
a_{1} a_{2} \ldots a_{n} \odot b_{1} b_{2} \ldots b_{m}=a_{1}
$$

$a_{2} \ldots a_{n} b_{1} b_{2} \ldots b_{m}$.

It may be noted that strings can be concatenated by giving another string. If $\alpha$ and $\beta$ be strings in $\Sigma^{*}$, then $\alpha \odot \beta$ denotes the concatenation of $\alpha$ and $\beta$, that is, the string formed by making a copy of $\alpha$ and $\beta$

$$
\text { If } \alpha=011011 \text { and } \beta=1110 \text {, then }
$$

$\alpha \odot \beta=0110111110$ and

\section{$\beta \odot \alpha=1110011011$}

Note that $\alpha \odot \beta \neq \beta \odot \alpha$.

If $\varepsilon$ is the identity for concatenation, then for any string $\mathrm{w}$, the equation $\varepsilon \mathrm{w}=\mathrm{w}=\mathrm{w} \varepsilon$ hold.

Definition 1.3: If $\mathrm{S}$ and $\mathrm{T} \in \Sigma^{*}$, then

$$
S \odot T=\{s t / s \in S, t \in T\} \text {. }
$$

If $\Sigma$ is an alphabet set, and $\mathrm{L} \subseteq \Sigma^{*}$, then $\mathrm{L}$ is a formal language over $\Sigma$.

Definition 1.4: The union of two languages $L$ and $\mathrm{M}$, denoted by $L \cup M$ is the set of strings that are in L or M or both.

Example : If $\mathrm{L}=\{01,11,10\}$ and $\mathrm{M}=\{\varepsilon, 11\}$, then

$$
L \cup M=\{\varepsilon, 01,10,11\}
$$

Formal languages are usually defined in any one of the three ways.

I. regular expression

II. standard automata and

III. Formal language system.
Regular expressions usually consists of an

$$
\begin{array}{ll}
\text { Alphabet set A1 } & \{\mathrm{a}, \mathrm{b}, \mathrm{c}\} \\
\text { Alphabet set A2 } & =\{0,1,2\}
\end{array}
$$

Language L1 = the set of all words about A1 = $\{\mathrm{a}, \mathrm{ab}, \mathrm{ac}, \mathrm{bc}, \mathrm{aab}, \mathrm{abc}, \ldots .$.

Language L2 $=$ the set of all words over A2 = $\{0,01,02,12,012, \ldots .$.$\} .$

Language L3 $=L 1 \cup l 2$

Language L4 $=\{a n / n$ is even $\}=\{a a$, aaaa $\ldots .$.

Language L5 $=\{a n b n / n$ is natural $\}=$ $\{a b, a a b, a a b b \ldots\}$

Language L6 $=\{a n / n$ is odd $\}=\{a, a a a, \ldots . .$.

Languages can also be defined by any kind of automaton, like a Turing machine. Note that any automata or machine $\mathrm{M}$ operating on an alphabet set a can produce a perfectly valid language L. To generate a sentence from the list of Languages mentioned above is of great importance. To generate a sentence we use language $\mathrm{L}_{\mathrm{i}}$. To complete a sentence, we use terminal symbols like $\left\{., L_{1}, L_{2}, L_{1} \cup L_{2}\right\}$. We use the metrics from Viswanadh. K et. al [9] to simplify this paper.

A fuzzy set is a generalized set to which objects can belong with various degrees of membership over the interval $[0,1]$. For fuzzy sets, we refer to the recent study by Kasi Viswanadh [4]. It deals with uncertainty and fuzziness arising from interrelated humanistic rights of phenomena such as subjectivity, thinking reasoning, cognition, and perception. A necessary and sufficient condition for the existence of $\Psi$ bounded solutions for linear system of differential equations are established in [5] and a new approach to the construction of a transition matrix are established in [10]. Qualitative properties of first order systems established in $[6,7,8]$ are used as a tool to establish our existence and uniqueness criteria for three-point boundary value problems on time scales This paper makes use of the results established by Kasi Viswanadh V. Kanuri et.al $[3,11]$ are used as a tool to establish our main results. For results on initial value problems associated with First Order Fuzzy Difference Systems, we refer to $\mathrm{Y}$. $\mathrm{Wu}$ et.al in [12]. The premise of the fuzzy set theory is that the key elements human reasoning process are not members 
but labels of fussy sets. In formal languages we define, the alpha level sets $[\mathrm{u}]^{\alpha}$ as

$$
\left[\mathrm{u}^{(\alpha)}\right]=\mathrm{u}_{1}{ }^{(\alpha)} \mathrm{u}_{2}{ }^{(\alpha)} \ldots \mathrm{u}_{\mathrm{n}}{ }^{(\alpha)} \text {, }
$$

Where $\alpha \in[0,1]$. The degree of membership is specified by a number between 1 (full membership) and 0 (full non-membership). An ordinary set is special case fuzzy set, where the degree of membership is either 0 or 1 . By virtue of fuzziness human concepts like small, big, poor, rich, old, very old, normal and beautiful can be translated into a form of usable by computers.

The most classic theory of automata is reflected in cell biology. The rules are defined as follows:

i. If all the eight cells surrounding the current one are checked, the death arises if the count is less than two.

ii. If the current cell is off and the count is exactly three, then the birth is certain.

iii. If the count of the surrounding eight cells is exactly 2 , then the survival rate is more.

Like any manifestation of the theory of automata, the game of life can be defined using extremely simple and concise rules. In addition to the species level complexity illustrated by individual organism can be explained using the theory of automata. An organism might be complex in its full form, but examining constituent parts reveals consistency, symmetry and pattern analysis. Using the idea of the theory of formal languages and automata, we discuss various biological models and set of mathematical rules to analyze the behavior of a) Glucose and Insulin interaction and b) prey-predator model.

The question normally arises is that given any biological attribute, whether or not, we can stimulate an abstract machine and reduce the mathematical model into a more meaningful level of simplicity.

It is interesting to note that all bio-mathematical problems can be formulated within a formal system and can be allowed the mechanical creation and can be checked in a formal way.

\section{Prey-predator equation:}

The Lotka-volterra equations are also known as prey-predator equations. If $\mathrm{x}(\mathrm{t})$ denote preypopulation at time $\mathrm{t}$ and $\mathrm{y}(\mathrm{t})$ denote predator population at the same time $t$, then the system of non-linear biological equations when they interact with each other is given by

$$
\begin{aligned}
& \frac{d x}{d t}=\alpha x-\beta x y \\
& \frac{d y}{d t}=-\gamma y+\delta x y
\end{aligned}
$$

Where $\alpha, \beta, \gamma$, and $\delta$ are all positive constants. The Lotka-Voltena equations assumes a number of assumptions as follows:

1. The prey-population finds ample food at all times.

2. The food supply of the predator population depends entirely on the size of the preppopulation.

3. During the process the environment does not change in favor of one species and genetic adaptation is in inconsequential

4. The rate of change of population is proportional to its size.

5. Predators have limited appetite.

In the first equation,

$$
\frac{d x}{d t}=\alpha x-\beta x y \text {, }
$$

we observe that in the absence of predator population the prey population increases without bound and the interaction of these two populations is harmful to the prey-population and hence $(-\beta)$ is taken in the above equation.

The second equation refers to predator population

$$
\frac{d y}{d t}=-\gamma y+\delta x y
$$

The interaction of prey and predator specifies is beneficial to the growth of predator population and hence a +ve sign $\delta$ is taken. In the absence of prey population $(\mathrm{x}(\mathrm{t})=0)$, the predator population goes on decreasing.

The Lotka- Voltena system of equations is an example of a kolmogrov model, which is more general frame work that can model the dynamics of pre-predator interaction, disease and mutation, and economic model. The Jacobean matrix of the preypredator model is

$$
I(x, y)=\left[\begin{array}{lr}
\alpha-\beta y & -\beta x \\
\delta y & \delta x-\gamma
\end{array}\right]
$$

Obviously $(0,0)$ is an equilibrium point at $J(0,0)$, we have 


$$
I(0,0)=\left[\begin{array}{rr}
\alpha & 0 \\
0 & -\gamma
\end{array}\right]
$$

The eigenvalues of the matrix are

$\lambda_{1}=\alpha, \quad \lambda_{2}=-\gamma$

In this model $\alpha$ and $\gamma$ are always greater then 0 , and hence the fixed fix point at the origin is a saddle point.

If the level of prey-population increase beyond a threshold value, then the survival rate decreases due to limited resources of food.

If the level of predator population increases beyond a threshold value, then predator population decreases as they depend on prey population.

Suppose, there are two species of animals, a rat (prey population) and a cat (predator population). If the initial conditions are 80 rats and 40 cats, then we can plot the graph between the two species over time.

If all the eigenvalues of the system are non-positive then the prey-predator system is unstable condition. If both the eigenvalues of the system are positive, the system collapses after a limited time. If $\alpha>0$ and $\gamma<0$, then the system survives.

Finally we, conclude this paper with a classical problem on mathematics. $\sqrt{2}$ is not rational in the terminology of formal languages.

We are now in a position to present the detailed algorithm to compute closest vector point and this closest vector is the best least square solution of the system of equations

$\mathrm{DC}=\mathrm{f}$.

\section{Algorithm: CLOSEST VECTOR POINT (D,C).}

Input: A lattice point $\hat{C} \in \Lambda(D)$ the closest to $C$.

Step 1: Let $R_{2}=\omega D$ where $\omega$ is an $(m \times n)$ matrix with integer entries and det $\omega= \pm 1$

Step 2: Compute an $(m \times n)$ orthogonal matrix $\mathrm{Q}$ with orthonormal columns such that

$$
\mathrm{R}_{2}=\mathrm{R}_{3} \mathrm{Q} \text {, where } \mathrm{R}_{3} \text { is an }(\mathrm{m} \times \mathrm{n})
$$

lower- triangular matrix with all the diagonal elements greater then 0 .

Step 3: Let $H_{3}:=R_{2}^{-1}$
Step 4: Let $x_{3}:=C Q^{T}$

Step 5: Let $\widehat{U_{3}}:=\operatorname{DECODE}\left(R_{3}, C_{3}\right)$

Step 6: Return $\hat{C}:=\widehat{U_{3}} R_{2}$

Note that step1 is a basic reduction. If no basic reduction is needed, we can take $\omega$ as a unit matrix. Further, the speed and numerical stability of the search can be improved significantly, if proper search is made. Step 2 implies rotation and reflection of $\mathrm{R}_{2}$ into lower triangular form. The usual method adopted to achieve this is presented below as an algorithm.

Result 4.1: Let $D$ be an ( $r \times s)$ given matrix with rank $\mathrm{p} \leq \min \{\mathrm{r}, \mathrm{s}\}$. Then there exists a factorization

$\mathrm{DP}=\mathrm{QR}$

with the following properties:

i) $\mathrm{P}$ is an $(s \times s)$ permutation matrix with first $\mathrm{P}$ columns of DP form a basis of $\operatorname{Im}(\mathrm{D})$.

ii)Q is an ( $r \times p)$ matrix with orthonormal columns and $\mathrm{R}$ is $\mathrm{a}(\mathrm{p} \times \mathrm{s})$ upper trapezoidal matrix of the form $\mathrm{R}=\left[\mathrm{R}_{1}, \mathrm{R}_{2}\right]$, where $\mathrm{R}_{1}$ is an non-singular $(\mathrm{p} \times \mathrm{p})$ upper triangular matrix and $\mathrm{R}$ is a ( $\times \mathrm{s}-\mathrm{p})$ matrix.

\section{References}

[1.] E.V. Krishna Murthy \& S.S.Sen, theory of computer Science Narosa Publishing House , 1987.

[2.] Anderson James, Automata theory with modern applications, Cambridge University Press, 2006

[3.] Kasi Viswanadh V. Kanuri, K. N. Murty, "Three-Point boundary value problems associated with first order matrix difference system-existence and uniqueness via shortest and closest Lattice vector methods", Journal of Nonlinear Sciences and Applications, Volume 12, Issue 11, (2019) 720-727

[4.] Kasi Viswanadh V Kanuri, Existence Of $\Psi$ Bounded Solutions For Fuzzy Dynamical Systems On Time Scales, International Journal of Scientific \& Engineering Research, 2020, Vol. 11, No. 5, 613--624.

[5.] Kasi Viswanadh V. Kanuri, R. Suryanarayana, K. N. Murty, Existence of $\Psi$-bounded solutions for linear differential systems on time scales, Journal 
of Mathematics and Computer Science, 20 (2020), no. 1, 1--13.

[6.] Murty, K. N., Andreou, S., Viswanadh, K. V. K., Qualitative properties of general first order matrix difference systems. Nonlinear Studies, 16(2009), no. 4, 359-370.

[7.] K. N. Murty, V. V. S. S. S. Balaram, K.V. K. Viswanadh, "Solution of Kronecker Product Initial Value Problems Associated with First Order Difference System via Tensor-based Hardness of the Shortest Vector Problem", Electronic Modeling, vol. 6, p: 19-33 (2008).

[8.] K.N. Murty, K.V.K. Viswanadh, P. Ramesh, Yan Wu, "Qualitative properties of a system of differential equation involving Kronecker product of matrices", Nonlinear Studies, Vol 20, No: 3, P: 459--467 (2013)

[9.] K.N. Murty, Yan Wu, Viswanadh V. Kanuri, "Metrics that suit for dichotomy, well conditioning of object oriented design", Nonlinear Studies, Vol 18, No: 4, P: 621-637 (2013)
[10.] Kasi Viswanadh V Kanuri, Y. Wu, K.N. Murty, "Existence of $(\Phi \otimes \Psi)$ bounded solution of linear first order Kronecker product of system of differential equations", International Journal of Science and Engineering Research, 2020, Vol 11, No. 6, P: 721--730.

[11.] Kasi Viswanadh V. Kanuri, K. N. Murty, P. Sailaja, "A New Approach to The Construction of a Transition Matrix with Several Applications to Control System Theory", AIP Conference Proceedings, ICMM-757, December 2019 (In Press).

[12.] Y. Wu, D.L. Nethi, K.N.Murty, Initial Value Problems Associated With First Order Fuzzy Difference System -Existence And Uniqueness, International Journal of Recent Scientific Research. 11 (2020), no. 3, 37846--37848.

[13.] A.J Wilson .On Varma's Prey-predator problem bull mate biology Vol 42 PP 5996001980.

[14.] L.A.Zadeh, Fuzzy sets and systems, Inf. Control 8,338-353, 1965. 